\title{
ON SINGULAR ABREU EQUATIONS IN HIGHER DIMENSIONS
}

\author{
NAM Q. LE
}

\begin{abstract}
We study the solvability of the second boundary value problem of a class of highly singular, fully nonlinear fourth order equations of Abreu type in higher dimensions under either a smallness condition or radial symmetry.
\end{abstract}

\section{Introduction AND STATEMENTS OF THE MAIN RESUlts}

In this paper, which is a sequel to [6], we study the solvability of the second boundary value problem of a class of highly singular, fully nonlinear fourth order equations of Abreu type for a uniformly convex function $u$ :

$$
\left\{\begin{aligned}
\sum_{i, j=1}^{n} U^{i j} w_{i j} & =F\left(\cdot, u, D u, D^{2} u\right) & & \text { in } \Omega, \\
w & =\left(\operatorname{det} D^{2} u\right)^{-1} & & \text { in } \Omega, \\
u & =\varphi & & \text { on } \partial \Omega, \\
w & =\psi & & \text { on } \partial \Omega .
\end{aligned}\right.
$$

Here and throughout, $U=\left(U^{i j}\right)_{1 \leq i, j \leq n}$ is the cofactor matrix of the Hessian matrix $D^{2} u=$ $\left(u_{i j}\right)_{1 \leq i, j \leq n} \equiv\left(\frac{\partial^{2} u}{\partial x_{i} \partial x_{j}}\right)_{1 \leq i, j \leq n} ; \varphi \in C^{3,1}(\bar{\Omega}), \psi \in C^{1,1}(\bar{\Omega})$ with $\inf _{\partial \Omega} \psi>0$. The left hand side of (1.1) usually appears in Abreu's equation [1] in the problem of finding Kähler metrics of constant scalar curvature in complex geometry.

This type of equation arises from studying approximation of convex functionals such as the Rochet-Choné model in product line design [9] whose Lagrangians depend on the gradient variable, subject to a convexity constraint. Carlier-Radice [2] studied equation of the type (1.1) when $F$ does not depend on the Hessian variable. When the function $F$ depends on the Hessian variable, (1.1) was studied in [6] in two dimensions, including the case $F=-\Delta u$.

Note that (1.1) consists of a Monge-Ampère equation for $u$ in the form of $\operatorname{det} D^{2} u=w^{-1}$ and a linearized Monge-Ampère equation for $w$ in the form of

$$
\sum_{i, j=1}^{n} U^{i j} w_{i j}=F\left(\cdot, u, D u, D^{2} u\right)
$$

because the coefficient matrix $\left(U^{i j}\right)$ comes from linearization of the Monge-Ampère operator:

$$
U^{i j}=\frac{\partial \operatorname{det} D^{2} u}{\partial u_{i j}}
$$

2010 Mathematics Subject Classification. 35J40, 35B65, 35J96.

Key words and phrases. Singular Abreu equation, second boundary value problem, Monge-Ampère equation, linearized Monge-Ampère equation.

The research of the author was supported in part by the National Science Foundation under grant DMS-1764248. 
The solvability of second boundary problems such as (1.1) is usually established via a priori fourth order derivative estimates and degree theory. Two of the key ingredients for the a priori estimates are to establish (see [6]):

(i) positive lower and upper bounds for the Hessian determinant $\operatorname{det} D^{2} u$; and

(ii) global Hölder continuity for $w$ from global Hölder continuity of the linearized MongeAmpère equation with right hand side having low integrability.

By Theorem 1.7 in combination with Lemma 1.5 in [8], any integrability more than $n / 2$ right hand side of the linearized Monge-Ampère equation suffices for the global Hölder continuity and $n / 2$ is the precise threshold. The reason to restrict the analysis in [6] to two dimensions even for the simple case $F=-\Delta u$ is that either $\Delta u$ is just a measure or it belongs to $\Delta u \in L^{1+\varepsilon_{0}}(\Omega)$ where $\varepsilon_{0}>0$ can be arbitrary small. The condition $n / 2<1+\varepsilon_{0}$ with small $\varepsilon_{0}$ naturally leads to $n=2$.

In all dimensions, once we have the global Hölder continuity of $w$ together with the lower and upper bounds on $\operatorname{det} D^{2} u$, we can apply the global $C^{2, \alpha}$ estimates for the Monge-Ampère equation in [10, 13] to conclude that $u \in C^{2, \alpha}(\bar{\Omega})$. We update this information to $U^{i j} w_{i j}=F\left(\cdot, u, D u, D^{2} u\right)$ to have a second order uniformly elliptic equation for $w$ with global Hölder continuous coefficients and bounded right hand side. This gives second order derivatives estimates for $w$. Now, fourth order derivative estimates for $u$ easily follows.

In this paper, we consider the higher dimensional case of (1.1), focusing on the right-hand side being of $p$-Laplacian type. In this case, the first two equations of (1.1) arise as the Euler-Lagrange equation of the convex functional

$$
J_{p}(u):=\int_{\Omega}\left(\frac{|D u|^{p}}{p}-\log \operatorname{det} D^{2} u\right) d x
$$

When $p=2$, that is, (1.1) with $F=-\Delta u$, the a priori lower bound on $\operatorname{det} D^{2} u$ in [6] breaks down when $n \geq 3$.

Key to this analysis in [6] is the fact that trace $(U)=\Delta u$ in dimensions $\mathrm{n}=2$. With this crucial fact, one can use

$$
U^{i j}\left(w+\frac{1}{2}|x|^{2}\right)_{i j}=-\Delta u+\operatorname{trace}(U) \geq 0
$$

and then applying the maximum principle to conclude that $w+\frac{1}{2}|x|^{2}$ attains its maximum on $\partial \Omega$ from which the upper bound on $w$ follows which in turn implies the desired lower bound on $\operatorname{det} D^{2} u$.

If $n \geq 3$, the ratio $\frac{\operatorname{trace}(U)}{\Delta u}$ can be in general as small as we want; in fact, this is the case, say, when one eigenvalue of $\stackrel{\Delta}{D}^{2} u$ is 1 while all other $n-1$ eigenvalues are a small constant.

Here, we use a new technique to solve (1.1) when $F=-\gamma \operatorname{div}\left(|D u|^{p-2} D u\right)$ where $p \geq 2$ and $\gamma$ is small. More generally, our main result states as follows.

Theorem 1.1. Assume $n \geq 3$. Let $\Omega$ be an open, smooth, bounded and uniformly convex domain in $\mathbb{R}^{n}$. Let $\psi \in C^{2, \beta}(\bar{\Omega})$ with $\inf _{\partial \Omega} \psi>0$ and let $\varphi \in C^{4, \beta}(\bar{\Omega})$ where $\beta \in(0,1)$. Let $F(\cdot, z, \mathbf{p}, \mathbf{r})$ : $\bar{\Omega} \times \mathbb{R} \times \mathbb{R}^{n} \times \mathbb{R}^{n \times n}$ be a smooth function such that:

(i) it maps compact subsets of $\bar{\Omega} \times \mathbb{R} \times \mathbb{R}^{n} \times \mathbb{R}^{n \times n}$ into compact subsets of $\mathbb{R}$ and

(ii) $F\left(x, u(x), D u(x), D^{2} u(x)\right) \leq 0$ in $\Omega$ for all $C^{2}$ convex function $u$. 
If $\gamma>0$ is a small constant depending only on $\beta, \varphi, \psi, n, F$ and $\Omega$, then there is a uniform convex solution $u \in C^{4, \beta}(\bar{\Omega})$ to the following second boundary value problem:

$$
\left\{\begin{aligned}
\sum_{i, j=1}^{n} U^{i j} w_{i j} & =\gamma F\left(\cdot, u, D u, D^{2} u\right) & & \text { in } \Omega, \\
w & =\left(\operatorname{det} D^{2} u\right)^{-1} & & \text { in } \Omega, \\
u & =\varphi & & \text { on } \partial \Omega, \\
w & =\psi & & \text { on } \partial \Omega .
\end{aligned}\right.
$$

The solution is unique provided that $F$ additionally satisfies

(1.4) $\int_{\Omega}\left[F\left(\cdot, u, D u, D^{2} u\right)-F\left(\cdot, v, D v, D^{2} v\right)\right](u-v) d x \geq 0$ for all $u, v \in C^{2}(\bar{\Omega})$ with $u=v$ on $\partial \Omega$.

Remark 1.2. It would be very interesting to remove the smallness of $\gamma$ in Theorem 1.1 ,

Our next result is concerned with radial solutions for $p$-Laplacian right hand side.

Theorem 1.3. Assume that $\Omega=B_{1}(0) \subset \mathbb{R}^{n}$ and let $\varphi$ and $\psi$ be constants with $\psi>0$. Let $p \in(1, \infty)$. Let $\beta=p-1$ if $p<2$ and $\beta \in(0,1)$ if $p \geq 2$. Let $f \in\{-1,1\}$. Consider the second boundary value problem:

$$
\left\{\begin{aligned}
\sum_{i, j=1}^{n} U^{i j} w_{i j} & =f \operatorname{div}\left(|D u|^{p-2} D u\right) & & \text { in } \Omega, \\
w & =\left(\operatorname{det} D^{2} u\right)^{-1} & & \text { in } \Omega, \\
u & =\varphi & & \text { on } \partial \Omega, \\
w & =\psi & & \text { on } \partial \Omega .
\end{aligned}\right.
$$

(i) Let $f=-1$. Then there is a unique radial, uniformly convex solution $u \in C^{3, \beta}(\bar{\Omega})$ to $(1.5)$.

(ii) Let $f=1$ and let $p \in(1, n]$. In the case $p=n$, we assume further that $\psi>\frac{1}{n}$. Then there is a unique radial, uniformly convex solution $u \in C^{3, \beta}(\bar{\Omega})$ to 1.5 ).

(iii) Let $f=1$ and let $p>n$. Suppose that $\psi \geq M(n, p)$ for some sufficiently large constant $M>0$. Then there is a radial, uniformly convex solution $u \in C^{3, \beta}(\bar{\Omega})$ to (1.5).

Remark 1.4. Regarding p-Laplacian right hand side, even in the two dimensions, the analysis in [6] left open the case $F=-\operatorname{div}\left(|D u|^{p-2} D u\right)$ when $p \in(1,2)$. The missing ingredient was the lower bound for $\operatorname{det} D^{2} u$ in the a priori estimates. If this is obtained, then one can use the recent result in [7] to establish the solvability of (1.1); see the proof of Theorem 1.3 in [7.

Remark 1.5. The size condition on $\psi$ in Theorem 1.3 (ii) is optimal. We can see this in two dimensions as follows. If $f \equiv 1, n=p=2$ and $0<\psi \leq 1 / 2$, then there are no uniformly convex solutions $u \in C^{4}(\bar{\Omega})$ to (1.5). Indeed, if such a uniformly convex solution $u$ exists then the first and the last equation of (1.5) implies that

$$
w(x)=\psi+\frac{1}{2}\left(|x|^{2}-1\right) .
$$

However, since $\psi \leq 1 / 2$, there is $x \in \Omega$ such that $w(x) \leq 0$, which is a contradiction to the uniform convexity of $u$ and $w=\left(\operatorname{det} D^{2} u\right)^{-1}$.

When $n=p=2$, we can remove the symmetry conditions in Theorem 1.3 , 
Proposition 1.6. Let $\Omega$ be an open, smooth, bounded and uniformly convex domain in $\mathbb{R}^{n}$ where $n=2$. Assume $f \geq 0$ and $f \in L^{\infty}(\Omega)$. Assume that $\varphi \in W^{4, q}(\Omega), \psi \in W^{2, q}(\Omega)$ where $q>n$ with

$$
\inf _{x \in \partial \Omega}\left(\psi(x)-\frac{\|f\|_{L^{\infty}(\Omega)}}{2}|x|^{2}\right)>0 .
$$

Then there is a uniform convex solution $u \in W^{4, q}(\Omega)$ to the following second boundary value problem:

$$
\left\{\begin{aligned}
\sum_{i, j=1}^{n} U^{i j} w_{i j} & =f \Delta u & & \text { in } \Omega, \\
w & =\left(\operatorname{det} D^{2} u\right)^{-1} & & \text { in } \Omega, \\
u & =\varphi & & \text { on } \partial \Omega, \\
w & =\psi & & \text { on } \partial \Omega .
\end{aligned}\right.
$$

If $f$ is a nonnegative constant, $\varphi \in C^{\infty}(\bar{\Omega})$, and $\psi \in C^{\infty}(\bar{\Omega})$ then there is a solution $u \in C^{\infty}(\bar{\Omega})$.

The key ingredient in the proof of Theorem 1.1 is the solvability and uniform estimates in $W^{4, p}(\Omega)$ for $p>n$ of (1.1) when

$$
F \sim-(\Delta u)^{\frac{1}{n-1}}\left(\operatorname{det} D^{2} u\right)^{\frac{n-2}{n-1}}
$$

which reduces to $F \sim-\Delta u$ in two dimensions. This result, and its slightly more general version in Proposition 1.7, can be of independent interest.

Proposition 1.7. Let $\Omega$ be an open, smooth, bounded and uniformly convex domain in $\mathbb{R}^{n}$. Assume that $\varphi \in W^{4, q}(\Omega), \psi \in W^{2, q}(\Omega)$ with $\inf _{\partial \Omega} \psi>0$ where $q>n$. Let $k \in\{1, \cdots, n-1\}$. Assume that $0 \leq f, g \leq 1$. Then there is a uniform convex solution $u \in W^{4, q}(\Omega)$ to the following second boundary value problem:

$$
\left\{\begin{aligned}
\sum_{i, j=1}^{n} U^{i j} w_{i j} & =-(\Delta u)^{\frac{1}{n-1}}\left(\operatorname{det} D^{2} u\right)^{\frac{n-2}{n-1}} f-\left[S_{k}\left(D^{2} u\right)\right]^{\frac{1}{k(n-1)}}\left(\operatorname{det} D^{2} u\right)^{\frac{n-2}{n-1}} g & & \text { in } \Omega, \\
w & =\left(\operatorname{det} D^{2} u\right)^{-1} & & \text { in } \Omega, \\
u & =\varphi & & \text { on } \partial \Omega, \\
w & =\psi & & \text { on } \partial \Omega .
\end{aligned}\right.
$$

If $f \equiv 1$ and $g \equiv 1, \varphi \in C^{4, \beta}(\bar{\Omega})$, and $\psi \in C^{2, \beta}(\bar{\Omega})$ then there is a solution $u \in C^{4, \beta}(\bar{\Omega})$.

In Proposition 1.7 and what follows, for a symmetric $n \times n$ matrix $A$ with eigenvalues $\lambda_{1}, \cdots, \lambda_{n}$, let us denote its elementary symmetric functions $S_{k}(A)$ where $k=0,1, \cdots, n$ by

$$
S_{0}(A)=1, S_{k}(A)=\sum_{1 \leq i_{1}<\cdots<i_{k} \leq n} \lambda_{i_{1}} \cdots \lambda_{i_{k}}(k \geq 1) .
$$

The rest of the paper is devoted to proving Theorems 1.1 and 1.3, and Propositions 1.6 and 1.7.

\section{Proofs of the MAIN Results}

In this section, we prove Theorems 1.1 and 1.3, and Propositions 1.6 and 1.7, As in 6], it suffices to prove appropriate fourth order derivative a priori estimates.

For certain fixed parameters $\beta$ (in Theorem 1.1), $p$ (in Theorem 1.3) and $k, q$ (in Propositions 1.6 and 1.7), we call a positive constant universal if it depends only on $n, \Omega, \psi, \varphi$ and those fixed parameters. We use $c, C, C_{1}, C_{2}, \cdots$, to denote universal constants and their values may change from line to line. 
Proof of Proposition 1.7. For simplicity, we denote

$$
F(x)=-(\Delta u(x))^{\frac{1}{n-1}}\left(\operatorname{det} D^{2} u(x)\right)^{\frac{n-2}{n-1}} f(x)-\left[S_{k}\left(D^{2} u(x)\right)\right]^{\frac{1}{k(n-1)}}\left(\operatorname{det} D^{2} u(x)\right)^{\frac{n-2}{n-1}} g(x) .
$$

We establish a priori estimates for a solution $u \in W^{4, q}(\Omega)$. Since $U^{i j} w_{i j} \leq 0$, by the maximum principle, the function $w$ attains its minimum value on the boundary $\partial \Omega$. Thus

$$
w \geq \inf _{\partial \Omega} \psi:=C_{1}>0 .
$$

On the other hand, we note that for each $k \in\{1, \cdots, n-1\}$,

$$
\Delta u \geq\left[S_{k}\left(D^{2} u\right)\right]^{\frac{1}{k}}
$$

and furthermore,

$$
\operatorname{trace}\left(U^{i j}\right)=S_{n-1}\left(D^{2} u\right) \geq(\Delta u)^{\frac{1}{n-1}}\left(\operatorname{det} D^{2} u\right)^{\frac{n-2}{n-1}} .
$$

Indeed, (2.2) is equivalent to $\left(\operatorname{det} D^{2} u\right) \operatorname{trace}\left(D^{2} u^{-1}\right) \geq(\Delta u)^{\frac{1}{n-1}}\left(\operatorname{det} D^{2} u\right)^{\frac{n-2}{n-1}}$, or

$$
\left[\operatorname{Trace}\left(D^{2} u^{-1}\right)\right]^{n-1} \geq \frac{\Delta u}{\operatorname{det} D^{2} u} .
$$

Let $\lambda_{1}, \cdots, \lambda_{n}$ be eigenvalues of $D^{2} u$. Then (2.3) reduces to

$$
\left(\sum_{j=1}^{n} \frac{1}{\lambda_{j}}\right)^{n-1} \geq \frac{\sum_{i=1}^{n} \lambda_{i}}{\prod_{i=1}^{n} \lambda_{i}}=\sum_{i=1}^{n} \prod_{j \neq i}^{n} \frac{1}{\lambda_{j}} .
$$

This is obvious by the expansion of the left hand side.

It follows from (2.1) and (2.2) and $0 \leq f, g \leq 1$ that

$$
U^{i j}\left(w+|x|^{2}\right)_{i j} \geq 0 \text {. }
$$

By the maximum principle, the function $w+|x|^{2}$ attains its maximum value on the boundary $\partial \Omega$. Thus

$$
w+|x|^{2} \leq \max _{\partial \Omega}\left(\psi+|x|^{2}\right) \leq C_{2}<\infty .
$$

Therefore $w \leq C_{2}$. As a consequence,

$$
C_{1} \leq w \leq C_{2} .
$$

From the second equation of (1.8), we can find a universal constant $C>0$ such that

$$
C^{-1} \leq \operatorname{det} D^{2} u \leq C \text { in } \Omega .
$$

By constructing a suitable barrier, we find that $D u$ is universally bounded in $\bar{\Omega}$ :

$$
\|D u\|_{L^{\infty}(\Omega)} \leq C .
$$

From $\varphi \in W^{4, q}(\Omega)$ with $q>n$, we have $\varphi \in C^{3}(\bar{\Omega})$ by the Sobolev embedding theorem. By assumption, $\Omega$ is bounded, smooth and uniformly convex. From $u=\varphi$ on $\partial \Omega$ and (2.4), we can apply the global $W^{2,1+\varepsilon_{0}}$ estimates for the Monge-Ampère equation, which follow from the interior $W^{2,1+\varepsilon_{0}}$ estimates in De Philippis-Figalli-Savin [3] and Schmidt [12] and the global estimates in Savin [11] (see also [4, Theorem 5.3]), to conclude that

$$
\left\|D^{2} u\right\|_{L^{1+\varepsilon_{0}(\Omega)}} \leq C_{1}^{*}
$$

for some universal constants $\varepsilon_{0}>0$ and $C_{1}^{*}>0$.

Thus, from (2.6) and (2.1), we find that

$$
\|F\|_{L^{(n-1)\left(1+\varepsilon_{0}\right)(\Omega)}} \leq C_{3}
$$

for a universal constant $C_{3}>0$. Note that for all $n \geq 2$ and all $\varepsilon_{0}>0$,

$$
(n-1)\left(1+\varepsilon_{0}\right)>n / 2 \text {. }
$$


From $\psi \in W^{2, q}(\Omega)$ with $q>n$, we have $\psi \in C^{1}(\bar{\Omega})$ by the Sobolev embedding theorem. Now, we apply the global Hölder estimates for the linearized Monge-Ampère equation in [8, Theorem 1.7 and Lemma 1.5] to $U^{i j} w_{i j}=F$ in $\Omega$ with boundary value $w=\psi \in C^{1}(\partial \Omega)$ on $\partial \Omega$ to conclude that $w \in C^{\alpha}(\bar{\Omega})$ with

$$
\|w\|_{C^{\alpha}(\bar{\Omega})} \leq C\left(\|\psi\|_{C^{1}(\partial \Omega)}+\|F\|_{L^{(n-1)\left(1+\varepsilon_{0}\right)}(\Omega)}\right) \leq C_{4}
$$

for universal constants $\alpha \in(0,1)$ and $C_{4}>0$. Now, we note that $u$ solves the Monge-Ampère equation

$$
\operatorname{det} D^{2} u=w^{-1}
$$

with right hand side being in $C^{\alpha}(\bar{\Omega})$ and boundary value $\varphi \in C^{3}(\partial \Omega)$ on $\partial \Omega$. Therefore, by the global $C^{2, \alpha}$ estimates for the Monge-Ampère equation [13, 10], we have $u \in C^{2, \alpha}(\bar{\Omega})$ with universal estimates

$$
\|u\|_{C^{2, \alpha}(\bar{\Omega})} \leq C_{5} \text { and } C_{5}^{-1} I_{n} \leq D^{2} u \leq C_{5} I_{n} .
$$

Here and throughout, we use $I_{n}$ to denote the $n \times n$ identity matrix. As a consequence, the second order operator $U^{i j} \partial_{i j}$ is uniformly elliptic with Hölder continuous coefficients. Now, we observe from the definition of $F$ and (2.8) that

$$
\|F\|_{L^{\infty}(\Omega)} \leq C_{6} .
$$

Thus, from the equation $U^{i j} w_{i j}=F$ with boundary value $w=\psi$ where $\psi \in W^{2, q}(\Omega)$, we conclude that $w \in W^{2, q}(\Omega)$ and therefore $u \in W^{4, q}(\Omega)$ with universal estimate

$$
\|u\|_{W^{4, q}(\Omega)} \leq C_{7} \text {. }
$$

It remains to consider the case $f \equiv 1$ and $g \equiv 1, \varphi \in C^{4, \beta}(\bar{\Omega})$, and $\psi \in C^{2, \beta}(\bar{\Omega})$. In this case, we need to establish a priori estimates for $u \in C^{4, \beta}(\bar{\Omega})$. As above, instead of (2.9), we have

$$
\|F\|_{C^{\frac{\alpha}{n-1}(\bar{\Omega})}} \leq C_{7} .
$$

Thus, from the equation $U^{i j} w_{i j}=F$ with boundary value $w=\psi$ where $\psi \in C^{2, \beta}(\bar{\Omega})$, we conclude that $w \in C^{2, \gamma}(\bar{\Omega})$ where $\gamma:=\min \left\{\frac{\alpha}{n-1}, \beta\right\}$ and therefore $u \in C^{4, \gamma}(\bar{\Omega})$ with the universal estimate $\|u\|_{C^{4, \gamma}(\bar{\Omega})} \leq C_{8}$. With this estimate, we can improve (2.10) to

$$
\|F\|_{C^{\beta}(\bar{\Omega})} \leq C_{9} .
$$

As above, we find that $u \in C^{4, \beta}(\bar{\Omega})$ with the universal estimate $\|u\|_{C^{4, \beta}(\bar{\Omega})} \leq C_{10}$.

Proof of Theorem 1.1. Without loss of generality, we can assume that $\inf _{\partial \Omega} \psi=1$. We consider the following second boundary value problem for a uniformly convex function $u$ :

$$
\left\{\begin{aligned}
U^{i j} w_{i j} & =-(\Delta u)^{\frac{1}{n-1}}\left(\operatorname{det} D^{2} u\right)^{\frac{n-2}{n-1}} f_{\gamma}\left(\cdot, u, D u, D^{2} u\right) & & \text { in } \Omega, \\
w & =\left(\operatorname{det} D^{2} u\right)^{-1} & & \text { in } \Omega, \\
u & =\varphi & & \text { on } \partial \Omega, \\
w & =\psi & & \text { on } \partial \Omega .
\end{aligned}\right.
$$

for some $\gamma \in(0,1)$ to be chosen later, where

$$
f_{\gamma}\left(\cdot, u, D u, D^{2} u\right)=\min \left\{\frac{-\gamma F\left(\cdot, u, D u, D^{2} u\right)}{(\Delta u)^{\frac{1}{n-1}}\left(\operatorname{det} D^{2} u\right)^{\frac{n-2}{n-1}}}, 1\right\} .
$$

By our assumption (ii) on $F$, when $u$ is a $C^{2}$ convex function, we have $0 \leq f_{\gamma} \leq 1$. By Proposition 1.7 (with $g \equiv 0$ ), (2.12) has a solution $u \in W^{4, q}(\Omega)$ for all $q<\infty$. Thus, the first equation of (2.12) holds pointwise a.e. 
As in the proof of Proposition 1.7 (see (2.8)), we have the following a priori estimates

$$
\|u\|_{C^{2, \beta}(\bar{\Omega})} \leq C_{1} \text { and } C_{1}^{-1} I_{n} \leq D^{2} u \leq C_{1} I_{n}
$$

for some $C_{1}>0$ depending only on $\beta, \varphi, \psi, n$ and $\Omega$. Hence, using the assumption (i) on $F$, we find that

$$
\frac{-\gamma F\left(\cdot, u, D u, D^{2} u\right)}{(\Delta u)^{\frac{1}{n-1}}\left(\operatorname{det} D^{2} u\right)^{\frac{n-2}{n-1}}}<\frac{1}{2}
$$

if $\gamma>0$ is small, depending only on $\beta, \varphi, \psi, n, F$ and $\Omega$.

Thus, if $\gamma>0$ is small, depending only on $\beta, \varphi, \psi, n, F$ and $\Omega$, then

$$
f_{\gamma}=\min \left\{\frac{-\gamma F\left(\cdot, u, D u, D^{2} u\right)}{(\Delta u)^{\frac{1}{n-1}}\left(\operatorname{det} D^{2} u\right)^{\frac{n-2}{n-1}}}, 1\right\}=\frac{-\gamma F\left(\cdot, u, D u, D^{2} u\right)}{(\Delta u)^{\frac{1}{n-1}}\left(\operatorname{det} D^{2} u\right)^{\frac{n-2}{n-1}}}
$$

in $\Omega$ and hence the first equation of (2.12) becomes

$$
U^{i j} w_{i j}=\gamma F\left(\cdot, u, D u, D^{2} u\right)
$$

Using this equation together with (2.13) and $\varphi \in C^{4, \beta}(\bar{\Omega})$ and $\psi \in C^{2, \beta}(\bar{\Omega})$, we easily conclude $u \in C^{4, \beta}(\bar{\Omega})$. Thus, there is a uniform convex solution $u \in C^{4, \beta}(\bar{\Omega})$ to $(\underline{1.3})$.

Assume now $F$ additionally satisfies (1.4). Then arguing as in the proof of [6, Lemma 4.5] replacing $f_{\delta}$ there by $\gamma F$, we obtain the uniqueness of $C^{4, \beta}(\bar{\Omega})$ solution to (1.3).

Remark 2.1. Clearly, Theorem 1.1 and its proof apply to dimensions $n=2$.

Proof of Proposition [1.6. We stablish a priori estimates for a solution $u \in W^{4, q}(\Omega)$ to (1.7). As in the proof of Proposition 1.7, it suffices to obtain the lower and upper bounds on $\operatorname{det} D^{2} u$.

Observe that

$$
U^{i j} w_{i j}=f \Delta u \geq 0 .
$$

By the maximum principle, the function $w$ attains its maximum value on the boundary $\partial \Omega$. Thus

$$
w \leq \sup _{\partial \Omega} \psi<\infty
$$

By the second equation of (1.7), this gives a bound from below for $\operatorname{det} D^{2} u$ :

$$
\operatorname{det} D^{2} u \geq C^{-1} \text {. }
$$

On the other hand, we have

$$
\sum_{i, j=1}^{2} U^{i j}\left(w-\frac{\|f\|_{L^{\infty}(\Omega)}}{2}|x|^{2}\right)_{i j}=\left(f-\|f\|_{L^{\infty}(\Omega)}\right) \Delta u \leq 0 .
$$

By the maximum principle, the function $w-\frac{\|f\|_{L^{\infty}(\Omega)}}{2}|x|^{2}$ attains its minimum value on the boundary $\partial \Omega$. Thus, using (1.6), we find that

$$
w-\frac{\|f\|_{L^{\infty}(\Omega)}}{2}|x|^{2} \geq \inf _{x \in \partial \Omega}\left(w(x)-\frac{\|f\|_{L^{\infty}(\Omega)}}{2}|x|^{2}\right)>0 .
$$

This gives a positive lower bound for $w$, that is, $w \geq C^{-1}>0$. Using the second equation of (1.7), we obtain a bound from above for $\operatorname{det} D^{2} u$ :

$$
\operatorname{det} D^{2} u \leq C \text {. }
$$


Proof of Theorem 1.3. Recall that $f \in\{-1,1\}$ and $\beta=p-1$ if $1<p<2$ and $\beta \in(0,1)$ if $p \geq 2$.

We first observe the following reduction of smoothness without any symmetry assumptions. Suppose that one has a uniformly convex solution $u \in C^{2}(\bar{\Omega})$ to (1.5) with positive lower and upper bounds on $\operatorname{det} D^{2} u$ :

$$
C^{-1} \leq \operatorname{det} D^{2} u \leq C
$$

for some $C>0$ and such that $w \in C^{\beta}(\bar{\Omega})$, then $u \in C^{3, \beta}(\bar{\Omega})$. Indeed, using (2.14) together with the global $C^{2, \alpha}$ estimates [13, 10] for the Monge-Ampère equation $\operatorname{det} D^{2} u=w^{-1}$ with boundary data $\varphi \in C^{3,1}(\bar{\Omega})$ and right hand side $w^{-1} \in C^{\beta}(\bar{\Omega})$, we have $u \in C^{2, \beta}(\bar{\Omega})$ with estimates

$$
\|u\|_{C^{2, \beta}(\bar{\Omega})} \leq C_{1} \text { and } C_{1}^{-1} I_{n} \leq D^{2} u \leq C_{1} I_{n}
$$

As a consequence, the second order operator $U^{i j} \partial_{i j}$ is uniformly elliptic with Hölder continuous coefficients with exponent $\beta \in(0,1)$. Note that $|D u|^{p-2} D u$ is Hölder continuous with exponent $\beta$. Using the first equation of (1.5), we see that the $C^{1, \beta}(\bar{\Omega})$ estimates for $w$ follows from [5. Theorem 8.33]. Hence, we have the $C^{3, \beta}(\bar{\Omega})$ estimates for $u$.

Now, we look for radial, uniformly convex solutions $u \in C^{2}(\bar{\Omega})$ to (1.5). Assume that the convex function $u$ is of the form

$$
u(x)=v(r)
$$

where

$$
v:[0, \infty) \rightarrow \mathbb{R} \text { and } r=|x|
$$

Let us denote

$$
=\frac{d}{d r} \quad \text { and } g(r):=v^{\prime}(r)
$$

The requirement that $u \in C^{2}(\bar{\Omega})$ forces

$$
g(0)=v^{\prime}(0)=0 .
$$

The next reduction in the proof of our theorem is the following claim.

Claim. The existence of radial, uniformly convex solutions $u \in C^{2}(\bar{\Omega})$ to (1.5) with positive lower and upper bounds on $\operatorname{det} D^{2} u$ and a Holder continuous $w$ is equivalent to finding $g(1)>0$ satisfying the integral equation

$$
\int_{0}^{g(1)} e^{\frac{f}{p} s^{p}} s^{n-1} d s=\frac{1}{n \psi}\left(1+f \int_{0}^{g(1)} e^{\frac{f}{p} s^{p}} s^{p-1} d s\right) .
$$

To prove the claim, we compute

$$
\operatorname{det} D^{2} u=v^{\prime \prime}\left(\frac{v^{\prime}}{r}\right)^{n-1}, w=\left(\operatorname{det} D^{2} u\right)^{-1}=\frac{1}{v^{\prime \prime}}\left(\frac{r}{v^{\prime}}\right)^{n-1} \equiv W(r) .
$$

Since $D^{2} u$ and $\left(D^{2} u\right)^{-1}$ are similar to $\operatorname{diag}\left(v^{\prime \prime}, \frac{v^{\prime}}{r}, \cdots, \frac{v^{\prime}}{r}\right)$ and $\operatorname{diag}\left(\frac{1}{v^{\prime \prime}}, \frac{r}{v^{\prime}}, \cdots, \frac{r}{v^{\prime}}\right)$, we can compute

$$
U^{i j} w_{i j}=\frac{v^{\prime \prime}\left(v^{\prime}\right)^{n-1}}{r^{n-1}}\left(\frac{W^{\prime \prime}}{v^{\prime \prime}}+(n-1) \frac{W^{\prime}}{v^{\prime}}\right)=\frac{\left[W^{\prime}\left(v^{\prime}\right)^{n-1}\right]^{\prime}}{r^{n-1}} .
$$

Note that $v^{\prime \prime}$ and $v^{\prime}$ are all nonnegative. Therefore,

$$
0 \leq v^{\prime}(r) \leq v^{\prime}(1) \text { for all } 0 \leq r \leq 1 .
$$

On the other hand, we have

$$
\operatorname{div}\left(|D u|^{p-2} D u\right)=(p-1)\left(v^{\prime}\right)^{p-2} v^{\prime \prime}+\frac{n-1}{r}\left(v^{\prime}\right)^{p-1}=\frac{\left[\left(v^{\prime}\right)^{p-1} r^{n-1}\right]^{\prime}}{r^{n-1}} .
$$


The first equation of (1.5) gives

$$
\frac{\left[W^{\prime}\left(v^{\prime}\right)^{n-1}\right]^{\prime}}{r^{n-1}}=f \frac{\left[\left(v^{\prime}\right)^{p-1} r^{n-1}\right]^{\prime}}{r^{n-1}}
$$

which implies that, for some constant $C$

$$
W^{\prime}\left(v^{\prime}\right)^{n-1}=f\left(v^{\prime}\right)^{p-1} r^{n-1}+C .
$$

Since $v^{\prime}(0)=0$, we find that $C=0$. Thus

$$
W^{\prime}=f\left(v^{\prime}\right)^{p-1}\left(\frac{r}{v^{\prime}}\right)^{n-1}=f\left(v^{\prime}\right)^{p-1} v^{\prime \prime} W
$$

It follows that

$$
[\log W]^{\prime}=\left[\frac{f}{p}\left(v^{\prime}\right)^{p}\right]^{\prime}
$$

and hence, recalling $W(1)=\psi$,

$$
\log W(r)=\log W(1)+\frac{f}{p}\left[\left(v^{\prime}(r)\right)^{p}-\left(v^{\prime}(1)\right)^{p}\right]=\log \psi+\frac{f}{p}\left[\left(v^{\prime}(r)\right)^{p}-\left(v^{\prime}(1)\right)^{p}\right] .
$$

Therefore, in terms of $g=v^{\prime}$, we have after exponentiation,

$$
e^{\frac{f}{p}[g(r)]^{p}}[g(r)]^{n-1} g^{\prime}(r)=\frac{1}{\psi} e^{\frac{f}{p}[g(1)]^{p}} r^{n-1},
$$

which is equivalent to

$$
\int_{0}^{g(r)} e^{\frac{f}{p} s^{p}} s^{n-1} d s=\frac{1}{n \psi} e^{\frac{f}{p}[g(1)]^{p}} r^{n} .
$$

Clearly, (2.20) leads to a solution to (1.5) in terms of $g(1), n, p$ and $\psi$ provided $g(1)>0$ satisfies the compatibility condition at $r=1$ :

$$
\int_{0}^{g(1)} e^{\frac{f}{p} s^{p}} s^{n-1} d s=\frac{1}{n \psi} e^{\frac{f}{p}[g(1)]^{p}} .
$$

Because

$$
e^{\frac{f}{p}[g(1)]^{p}}=1+f \int_{0}^{g(1)} e^{\frac{f}{p} s^{p}} s^{p-1} d s,
$$

the compatibility condition (2.21) can be rewritten as in (2.16).

Assume that $g(1)=v^{\prime}(1)>0$ has already been found, in terms of $n, p$ and $\psi$. We now establish positive lower and upper bounds on $\operatorname{det} D^{2} u$ and that $w \in C^{\beta}(\bar{\Omega})$. Indeed, from $0 \leq g(r) \leq g(1)$, we can easily estimate

$$
e^{\frac{-1}{p}[g(1)]^{p}} \frac{[g(r)]^{n}}{n} \leq \int_{0}^{g(r)} e^{\frac{f}{p} s^{p}} s^{n-1} d s \leq e^{\frac{1}{p}[g(1)]^{p}} \frac{[g(r)]^{n}}{n} .
$$

Hence (2.20) gives

$$
C^{-1} r \leq g(r) \leq C r
$$

for some $C$ depends only on $g(1)>0, n, p$ and $\psi$. Thus, from (2.19), we find that $v^{\prime \prime}$ and $\frac{v^{\prime}(r)}{r}$ are bounded from below and above by positive constants. Therefore, we have positive lower and upper bounds on $\operatorname{det} D^{2} u=v^{\prime \prime}\left(\frac{v^{\prime}}{r}\right)^{n-1}$. Moreover, $v^{\prime}(r)=|D u(x)| \in C^{\alpha}(\bar{\Omega})$ for all $\alpha \in(0,1)$. Using (2.18), we also find that $W$, and hence $w$, is in $C^{\alpha}(\bar{\Omega})$. In particular, $w \in C^{\beta}(\bar{\Omega})$. 
We have reduced our theorem to the existence and uniqueness of $g(1)>0$ solving (2.16) which we now address.

(i) Recall that $f=-1$. Note that (2.16) becomes

$$
\int_{0}^{g(1)} e^{\frac{-1}{p} s^{p}} s^{n-1} d s=\frac{1}{n \psi}\left(1-\int_{0}^{g(1)} e^{\frac{-1}{p} s^{p}} s^{p-1} d s\right) .
$$

Clearly, there is a unique $g(1)>0$ solving the above integral equation. Hence, there is a unique radial, uniformly convex solution $u \in C^{3, \beta}(\bar{\Omega})$ to (1.5).

(ii) Recall that $f=1$ and $p \in(1, n]$. Note that (2.16) becomes

$$
H(g(1)=I(g(1))
$$

where

$$
H(t):=\int_{0}^{t} e^{\frac{1}{p} s^{p}} s^{n-1} d s \quad \text { and } I(t):=\frac{1}{n \psi}\left(1+\int_{0}^{t} e^{\frac{1}{p} s^{p}} s^{p-1} d s\right) \equiv \frac{1}{n \psi} e^{\frac{t^{p}}{p}} .
$$

Consider first the case $p=n$. Then

$$
H(t)=e^{\frac{t^{n}}{n}}-1 \quad \text { and } I(t)=\frac{1}{n \psi} e^{\frac{t^{n}}{n}} .
$$

Therefore, from (2.22) we find an explicit formula for $g(1)$ from the equation

$$
e^{\frac{1}{n}[g(1)]^{n}}=\frac{n \psi}{n \psi-1}
$$

showing that existence and uniqueness of a solution $g(1)>0$ to (2.16) when $\psi>\frac{1}{n}$. As a result, there is a unique radial, uniformly convex solution $u \in C^{3, \beta}(\bar{\Omega})$ to (1.5). Moreover, $\psi>\frac{1}{n}$ is also the optimal condition for the existence of a radial solution to (1.5).

Now we consider the case $p \in(1, n)$ and $\psi>0$. We show that (2.22) has a unique solution $g(1)>$ 0 and hence there is a unique radial, uniformly convex solution $u \in C^{3, \beta}(\bar{\Omega})$ to (1.5). Indeed, since $1<p<n$, the integrand of $H(t)$ grows faster than that of $I(t)$. Since $H(0)=0<I(0)=\frac{1}{n \psi}$, the function $H(t)$ will cross $I(t)$ for the first time from below at some point $t_{0}>0$. Thus $g(1)=t_{0}>0$ is a solution of (2.22). To show the uniqueness of $g(1)$, we show that if $t>t_{0}$ then $H(t)>I(t)$. Indeed, using the definition of $t_{0}$, we find that $H^{\prime}\left(t_{0}\right) \geq I^{\prime}\left(t_{0}\right)$. This means that

$$
e^{\frac{1}{p} t_{0}^{p}} t_{0}^{n-1} \geq \frac{1}{n \psi} e^{\frac{1}{p} t_{0}^{p}} t_{0}^{p-1}
$$

or, equivalently,

$$
t_{0}^{n-p} \geq \frac{1}{n \psi}
$$

Thus, if $s>t_{0}$ then $s^{n-p}>\frac{1}{n \psi}$, that is,

$$
e^{\frac{1}{p} s^{p}} s^{n-1}>\frac{1}{n \psi} e^{\frac{1}{p} s^{p}} s^{p-1}
$$

and hence, for any $t>t_{0}$, we have

$$
H(t)=H\left(t_{0}\right)+\int_{t_{0}}^{t} e^{\frac{1}{p} s^{p}} s^{n-1} d s>I\left(t_{0}\right)+\frac{1}{n \psi} \int_{t_{0}}^{t} e^{\frac{1}{p} s^{p}} s^{p-1} d s=I(t) .
$$

(iii) Recall that $f=1$ and $p>n$. Assume that $\psi \geq M(n, p):=1+\frac{e^{1 / p}}{n}\left(\int_{0}^{1} e^{\frac{1}{p} s^{p}} s^{n-1} d s\right)^{-1}$. Then, there is a solution $g(1)>0$ to (2.22) where $H$ and $I$ are defined as in (2.23). Indeed, in this case, we have $1>\frac{e^{1 / p}}{n \psi}[H(1)]^{-1}=\frac{I(1)}{H(1)}$. Therefore $I(1)<H(1)$ while $I(0)>H(0)$. Thus, (2.22) has a solution $g(1) \in(0,1)$. Consequently, there is a radial, uniformly convex solution $u \in C^{3, \beta}(\bar{\Omega})$ to (1.5). 
Remark 2.2. When $p>n$, radial solutions in Theorem 1.3 (iii) are not unique in general. This corresponds to multiple crossings of $H$ and $I$ defined in (2.23). For example, this is in fact the case of $n=2, p=4$ and $\psi=1$. We can plot the graphs of $H$ and $I$ using Maple to find that, on $[0,2]$, they cross twice at $t_{1} \in(1,6 / 5)$ and $t_{2} \in(3 / 2,2)$.

Acknowledgement. The author would like to thank Connor Mooney for critical comments on a previous version of this paper. The author also thanks the anonymous referee for his/her crucial comments and suggestions that help strengthen and simplify the proof of Theorem 1.3 ,

\section{REFERENCES}

[1] Abreu, M. Kähler geometry of toric varieties and extremal metrics. Int. J. Math. 9 (1998), no. 6, 641-651.

[2] Carlier, G.; Radice, T. Approximation of variational problems with a convexity constraint by PDEs of Abreu type. Calc. Var. Partial Differential Equations. 58 (2019), no. 5, Art. 170, 13 pp.

[3] De Philippis, G.; Figalli, A.; Savin, O. A note on interior $W^{2,1+\varepsilon}$ estimates for the Monge-Ampère equation. Math. Ann. 357 (2013), no. 1, 11-22.

[4] Figalli, A. The Monge-Ampère equation and its applications. Zurich Lectures in Advanced Mathematics. European Mathematical Society (EMS), Zürich, 2017.

[5] Gilbarg, D.; Trudinger, N.S. Elliptic partial differential equations of second order, Reprint of the 1998 edition. Classics in Mathematics. Springer-Verlag, Berlin, 2001.

[6] Le, N. Q. Singular Abreu equations and minimizers of convex functionals with a convexity constraint, arXiv:1811.02355 1, Comm. Pure Appl. Math., to appear.

[7] Le, N. Q. Global Hölder estimates for 2D linearized Monge-Ampère equations with right-hand side in divergence form, arXiv:1902.08113 [math.AP], preprint.

[8] Le, N. Q.; Nguyen, T. Global $W^{1, p}$ estimates for solutions to the linearized Monge-Ampère equations. J. Geom. Anal. 27 (2017), no. 3, 1751-1788.

[9] Rochet, J.-C.; Choné, P. Ironing, sweeping and multidimensional screening. Econometrica 66 (1998), no. 4, $783-826$.

[10] Savin, O. Pointwise $C^{2, \alpha}$ estimates at the boundary for the Monge-Ampère equation. J. Amer. Math. Soc. 26 (2013), no. 1, 63-99.

[11] Savin, O. Global $W^{2, p}$ estimates for the Monge-Ampère equation. Proc. Amer. Math. Soc. 141 (2013), no. 10, 3573-3578.

[12] Schmidt, T. $W^{2,1+\varepsilon}$ estimates for the Monge-Ampère equation. Adv. Math. 240 (2013), 672-689.

[13] Trudinger, N.S.; Wang, X.J. Boundary regularity for the Monge-Ampère and affine maximal surface equations. Ann. of Math. (2) 167 (2008), no. 3, 993-1028.

Department of Mathematics, Indiana University, Bloomington, 831 E 3Rd St, IN 47405, USA.

E-mail address: nqle@indiana.edu 